\title{
Charting nearby stellar systems: The intensity of Galactic cosmic rays for a sample of solar-type stars
}

\author{
D. Rodgers-Lee ${ }^{1 \star}$, A. A. Vidotto ${ }^{1}$ and A. L. Mesquita ${ }^{1}$, \\ 1 School of Physics, Trinity College Dublin, University of Dublin, College Green, Dublin 2, D02 PN40, Ireland
}

Accepted 2021 September 22. Received 2021 August 30; in original form 2021 June 14.

\begin{abstract}
Cosmic rays can penetrate planetary atmospheres driving the formation of prebiotic molecules, which are important for the origin of life. We calculate the Galactic cosmic ray fluxes in the habitable zone of five nearby, well-studied solar-type stars and at the orbits of 2 known exoplanets. We model the propagation of Galactic cosmic rays through the stellar winds using a combined 1.5D stellar wind and $1 \mathrm{D}$ cosmic ray transport model.

We find that the habitable zone of $61 \mathrm{Cyg}$ A has comparable Galactic cosmic ray fluxes to present-day Earth values. For the other four systems ( $\epsilon$ Eri, $\epsilon$ Ind, $\xi$ Boo B and $\pi^{1} \mathrm{UMa}$ ), the fluxes are orders of magnitude smaller than Earth values. Thus, it is unlikely that any as-of-yet undetected Earth-like planets in their habitable zones would receive a higher radiation dose than is received on Earth. $\epsilon$ Ind b, a Jupiter-like planet orbiting at $\sim 11$ au, receives higher Galactic cosmic ray fluxes than Earth. We find the suppression of Galactic cosmic rays is influenced by whether diffusion or advection dominates at $\mathrm{GeV}$ energies and at distances where the wind has reached its' terminal velocity. For advectively-dominated winds ( younger systems), varying the astrospheric size influences the suppression significantly. For diffusion-dominated systems ( older systems) the astrospheric size, and therefore knowledge of the ISM properties, are not very important. This reduces the Galactic cosmic ray flux uncertainties in the habitable zone for diffusion-dominated systems. Whether a system is advection- or diffusion-dominated can be determined from the stellar wind properties.
\end{abstract}

Key words: diffusion - cosmic rays - methods: numerical - stars: solar-type - stars: winds, outflows - planetary systems

\section{INTRODUCTION}

Galactic cosmic rays ionise and dissociate molecules as they penetrate exoplanetary atmospheres. This can lead to the production of prebiotic molecules (Dartnell 2011). It has also recently been suggested that cosmic rays left an imprint on the helicity of DNA via their secondary particles, which are on-average spin polarised (Globus \& Blandford 2020; Globus et al. 2021). Thus, Galactic cosmic rays may have been important for the origin of life on Earth and on exoplanets. A number of fingerprint ions, indicative of ionisation by energetic particles (i.e. cosmic rays and stellar energetic particles) in exoplanetary atmospheres, have recently been identified (such as $\mathrm{H}_{3} \mathrm{O}^{+}$, Helling \& Rimmer 2019; Barth et al. 2021). Emission from fingerprint ions may be detectable using transmission spectroscopy with the James Webb Space Telescope (JWST, Gardner et al. 2006). This will place limits on the chemical abundances of fingerprint ions, and thus, on the flux of energetic particles in exoplanetary atmospheres. In order to perform chemical modelling of the impact of Galactic cosmic rays on exoplanetary atmospheres (e.g., Grießmeier et al. 2015; Barth et al. 2021) that will be characterised with

^ E-mail: drodgers@tcd.ie
JWST, it is important to model Galactic cosmic ray propagation for different stellar systems.

The flux of Galactic cosmic rays reaching an exoplanet's orbit depends on two factors: the properties of the stellar wind and the Galactic cosmic ray spectrum in the interstellar medium (ISM). For individual stellar systems knowledge of the local ISM properties, combined with Lyman- $\alpha$ observations, can be used to constrain the star's mass-loss rate (e.g. Wood 2004; Wood et al. 2014, 2021). Additionally, the ISM properties surrounding a stellar system, combined with the stellar wind properties, determine the size of the astrosphere. $\gamma$-ray observations indicate that the Galactic cosmic ray spectrum in the ISM is similar throughout the Galaxy (with the exception of the Galactic centre, Aharonian et al. 2020). Thus, the local interstellar spectrum (LIS) of Galactic cosmic rays (constrained by Voyager 1 \& 2 observations outside of the heliosphere, Cummings et al. 2016; Stone et al. 2019 ) is often adopted as the spectrum for other stellar systems.

The LIS is suppressed in an energy-dependent way as the cosmic rays travel through a stellar wind. This was first studied in the context of the solar system (e.g. Parker 1965). The suppression of Galactic cosmic rays varies spatially within the solar system and temporally with the magnetic solar cycle 
(Potgieter 2013). These variations are collectively described as the modulation of Galactic cosmic rays and affect cosmic rays with $\lesssim 30 \mathrm{GeV}$ energies in the solar system.

Recent efforts have focused on the Galactic cosmic ray fluxes, assuming diffusive cosmic ray transport, for the evolving solar wind (relevant for the origin of life on Earth, Rodgers-Lee et al. 2020b) and for a number of nearby $M$ dwarf systems (Herbst et al. 2020; Mesquita et al. 2021b) because exoplanets orbiting $M$ dwarf are prime targets in the search for life in the Universe. However, rocky planets in the habitable zone of $\mathrm{K}$ and $\mathrm{G}$ dwarfs, more similar to our own Sun, are still of great interest.

In addition to Galactic cosmic rays, stellar energetic particles (also known as stellar cosmic rays) are another type of energetic particle that are important for exoplanetary atmospheres (Airapetian et al. 2016; Tabataba-Vakili et al. 2016; Chen et al. 2020; Rodgers-Lee et al. 2021). Stellar energetic particle fluxes should be largest at young ages when the star's protoplanetary disk is still present (e.g. Rab et al. 2017; Rodgers-Lee et al. 2017, 2020a). However, here we only focus on the Galactic cosmic ray fluxes.

In this paper, we study the modulation of Galactic cosmic rays for a sample of solar-type stars ( $K$ and $G$ dwarfs). The sample comprises of 5 nearby stellar systems $(<14.4 \mathrm{pc}$ away) that have both stellar magnetic field measurements and mass-loss rates derived from Lyman- $\alpha$ observations, that we use to construct well-constrained stellar wind models. We use the stellar wind properties as an input for the cosmic ray propagation model. Mesquita et al. (2021a) perform a complementary study considering nearby $\mathrm{M}$ dwarfs.

The paper is structured as follows: Section 2 introduces the sample of stars that we consider, Sections 3 and 4 briefly discuss the stellar wind and cosmic ray tranport models that are used. Section 5 discusses our results and finally, our conclusions are presented in Section 6.

\section{DESCRIPTION OF SAMPLE}

Our sample consists of $\mathrm{G}$ and $\mathrm{K}$ dwarf stars that have a stellar wind mass-loss rate measurement (derived from Lyman- $\alpha$ observations) and ISM velocities (from Wood et al. 2014, and references therein), in addition to an observed X-ray luminosity (from Vidotto et al. 2014, 2016) and a stellar magnetic field map (from Vidotto et al. 2014; Boro Saikia et al. 2016; Vidotto et al. 2016). These observed properties are important to construct well-constrained stellar wind models for individual stars which is discussed in Section 3. The resulting sample consists of 5 nearby stars: 61 Cyg A, $\epsilon$ Eri, $\epsilon$ Ind, $\pi^{1}$ UMa and $\xi$ Boo B. Four of the stars are within $\sim 7$ pc of Earth placing them close to, or within, the local interstellar cloud (Redfield \& Linsky 2000). $\pi^{1}$ UMa is slightly further away ( $\left.\sim 14.4 \mathrm{pc}\right)$. The stellar masses range from $0.66-1 M_{\odot}$ and the stellar rotation rates vary from $0.8-5.4 \Omega \odot$. Age estimates for the systems range from 0.4 to $6 \mathrm{Gyr}$. The stellar masses, radii and rotation rates for our sample are from Vidotto et al. (2014, 2016) and Feng et al. (2019). The average unsigned radial stellar surface magnetic field strength values range from 3.8 - $18 \mathrm{G}$ from magnetic maps presented in Vidotto et al. (2014, 2016) and Boro Saikia et al. (2016) using the Zeeman Doppler
Imaging (ZDI) technique ${ }^{1}$. Vidotto et al. (2014, 2016) and Boro Saikia et al. (2016) present the average squared stellar magnetic field strength $\left\langle B^{2}\right\rangle=\frac{1}{4 \pi} \int\left(B_{r}^{2}+B_{\theta}^{2}+B_{\varphi}^{2}\right) \sin \theta d \theta d \varphi$ or $\langle|B|\rangle$. We use $\left\langle\left|B_{r}\right|\right\rangle$ for our stellar wind model since stellar winds are thought to be launched from open magnetic field lines. The observationally constrained stellar properties are given in Table 1.

Two of the stars are known to host Jupiter-like planets, namely $\epsilon$ Eri b $\left(1.56 M_{\mathrm{J}}\right.$ orbiting at $3.39 \mathrm{au}$, Hatzes et al. 2000; Benedict et al. 2006; Mawet et al. 2019) and $\epsilon$ Ind b $\left(3.25 M_{\mathrm{J}}\right.$ orbiting at $11.55 \mathrm{au}$, Feng et al. 2019), detected via radial velocity observations. Recent high-contrast imaging at $10 \mu \mathrm{m}$ by Pathak et al. (2021) with VISIR were not able to directly detect these planets. $\epsilon$ Eri is still surrounded by a debris disk with an estimated age of $0.4 \mathrm{Gyr}$. They estimate that, had $\epsilon$ Ind been 0.7 Gyr old, they would have detected the planet and that their non-detection implies an older age for $\epsilon$ Ind (consistent with other age estimates of 3.7-5.7 Gyr, Feng et al. 2019). Additionally, it is possible that there are undetected rocky Earth-like planets residing in the habitable zones of the stars in our sample.

$\pi^{1} \mathrm{UMa}$ is a very interesting object as it is considered to be a young solar analogue $\left(0.5 \mathrm{Gyr}, 1 R_{\odot}, 1 M_{\odot}\right)$. In contrast to predictions from spin down models (Johnstone et al. 2015), Wood et al. (2014) inferred a less-than-solar mass-loss rate $(\dot{M})$ for $\pi^{1}$ UMa from Lyman- $\alpha$ observations. Wood et al. (2014) discuss how it could be possible that a higher massloss $\left(150 \dot{M}_{\odot}\right)$ for $\pi^{1} \mathrm{UMa}$ is consistent with the observations if the ISM properties were significantly different (i.e. more ionised and lower in density). $150 \dot{M}_{\odot}$ represents the massloss rate obtained if the relation between solar flares and coronal mass ejections is extrapolated to a star as young/active as $\pi^{1}$ UMa (Drake et al. 2013). This value should be viewed as an upper limit as it is unlikely that $\pi^{1} \mathrm{UMa}$ would have such a high mass-loss rate due to energetic arguments (Drake et al. 2013). Given the discussion surrounding the mass-loss rate for $\pi^{1}$ UMa we investigate both scenarios: $0.5 \dot{M}_{\odot}$ (assuming $n_{\mathrm{ISM}}(\mathrm{H} \mathrm{I})=0.16 \mathrm{~cm}^{-3}$ and $n_{\mathrm{ISM}}\left(\mathrm{H}^{+}\right)=0.08 \mathrm{~cm}^{-3}$ from Wood et al. 2014) and $150 \dot{M}_{\odot}$ (assuming $n_{\mathrm{ISM}}(\mathrm{HI})=$ $0.0024 \mathrm{~cm}^{-3}$ and $n_{\mathrm{ISM}}\left(\mathrm{H}^{+}\right)=0.0456 \mathrm{~cm}^{-3}$ from Wood et al. 2014). See Vidotto (2021) for a discussion of the mass-loss rate measurements for $\pi^{1} \mathrm{UMa}$.

\section{STELLAR WIND MODEL}

The stellar wind is modelled using solutions of the $1.5 \mathrm{D}$ analytic isothermal model of Weber \& Davis (1967), presented in Johnstone (2017). This model accounts for stellar rotation. The wind is launched from the hot corona where it overcomes the star's gravity. The stellar wind accelerates rapidly before reaching its terminal velocity. The stellar properties relevant for the model are the stellar mass $\left(M_{\star}\right)$, radius $\left(R_{\star}\right)$, rotation rate $\left(\Omega_{\star}\right)$, magnetic field strength $\left(B_{\star}\right), \dot{M}$ and temperature of the wind $\left(T_{\text {base }}\right) . \dot{M}$ is the only parameter that is varied for each of the stars in our sample. All of the inputs for the stellar wind model are constrained by observations and are shown in Table 1.

1 The years of the observations for 61 Cyg A, $\epsilon$ Eri, $\epsilon$ Ind, $\pi^{1} \mathrm{UMa}$ and $\xi$ Boo B are 2010, 2008, 2013, 2012 and 2007, respectively. 
The wind temperature is assumed to be the temperature at the base of the wind since an isothermal model is used. The coronal temperature, $T_{\text {cor }}$, can be estimated from stellar X-ray emission as $T_{\text {cor }}=0.11 F_{\mathrm{X}}^{0.26}$ (Johnstone \& Güdel 2015). $F_{\mathrm{X}}$ is the stellar x-ray flux $\left(\mathrm{erg} \mathrm{cm}^{-2} \mathrm{~s}^{-1}\right)$ where $F_{\mathrm{X}}=L_{\mathrm{X}} / 4 \pi R_{\star}^{2}$. The X-ray luminosities, $L_{\mathrm{X}}$, for the sample are from Vidotto et al. $(2014,2016)$ and are given here in Table 1. Ó Fionnagáin \& Vidotto (2018) relate coronal temperatures to base temperatures, $T_{\text {base }}$, by dividing coronal temperatures by a factor of 1.36 to match solar observations near the base of the wind and we follow a similar approach here. Our adopted wind temperatures are shown in Table 2.

Stellar winds from low-mass stars are generally very challenging to measure because of the low densities of the winds. Wood et al. $(2002,2005,2014)$ derive mass-loss rates for the stars in our sample from Lyman- $\alpha$ observations which constrain the total neutral hydrogen column density between us, the observer, and the star. Lyman- $\alpha$ emission from the star is absorbed by neutral hydrogen at the edge of the astrosphere, the ISM and the edge of the heliosphere before being observed at Earth. The ISM ram pressure $\left(P_{\mathrm{ISM}}\right)$, and therefore the stellar wind ram pressure $\left(P_{\text {ram }}\right)$, most compatible with the observations is then inferred using typical values for the ISM density surrounding the stellar system and the ISM velocity. $P_{\text {ram }}$ depends on the stellar wind velocity $(v)$ and $\dot{M}$, i.e. $P_{\text {ram }}=\rho v^{2}=\frac{\dot{M} v}{4 \pi r^{2}}$ using $\dot{M}=4 \pi r^{2} \rho v$, where $\rho$ is the stellar wind density. In Wood et al. (2002, 2005, 2014), a solar wind terminal velocity $\left(v_{\infty, \odot}\right)$ is used, i.e. $400 \mathrm{~km} \mathrm{~s}^{-1}$, to calculate $\dot{M}$ for all of the stars.

The mass loss-rates from Wood et al. (2002, 2005, 2014) are denoted by $\dot{M}_{\mathrm{W}}$ and are shown in Table 1 for clarity. However, the stellar wind terminal velocities for low-mass stars should vary from star to star due to different coronal temperatures, stellar masses and radii (Parker 1965; Weber \& Davis 1967). We adopt a different approach to Wood et al. (2002, $2005,2014)$ and drop the assumption that $v_{\infty}=v_{\infty, \odot}$ for all of the stars. We use $\dot{M}_{\mathrm{W}}$ and $v_{\infty, \odot}$ to calculate $P_{\text {ram }}$ at a given radius where the wind has reached its terminal velocity for each star. We then use the terminal velocities obtained from the analytic Weber-Davis stellar wind model and vary the mass-loss rate in the model $\left(\dot{M}_{\mathrm{WD}}\right)$ until we match $P_{\mathrm{ram}}$ at the same given radius (which is the same methodology as adopted by Holzwarth \& Jardine 2007). The terminal wind velocities obtained for our sample of stars are larger than $v_{\infty, \odot}=400 \mathrm{~km} \mathrm{~s}^{-1}$, as adopted by Wood et al. (2002, 2005, 2014). Thus, the values of $\dot{M}_{\mathrm{WD}}$ that we find are correspondingly lower (given in Table 2) in order to match $P_{\text {ram }}$.

The magnetic field and velocity of the stellar winds as a function of orbital distance are used in the cosmic ray transport model which is described in Section 4. The wind profiles used are shown in Figure A1, as well as a brief description of how the stellar wind model outputs are extrapolated for the cosmic ray model. $P_{\text {ram }}$ is used to calculate the size of the astrosphere for each star in Section 3.1. For comparison, we also model the solar wind. For this model $\left|B_{\odot}\right|=1.9 \mathrm{G}$ (solar minimum value from Vidotto et al. 2014) and $T_{\text {base }, \odot}=1.5 \mathrm{MK}$ (Ó Fionnagáin \& Vidotto 2018) is used resulting in $v_{\infty} \sim 560 \mathrm{~km} \mathrm{~s}^{-1}$. This value is larger than the value adopted by Wood et al. $(2002,2005,2014)$ but both are consistent with observations of the solar wind (McComas et al. 2008).

\subsection{Astrospheric size}

The size of a star's astrosphere, $R_{\text {ast }}$, represents the star's dynamic sphere of influence and is determined by the balance of $P_{\text {ram }}$ and $P_{\mathrm{ISM}}$. This can be expressed as

$$
R_{\text {ast }}=\left(\frac{P_{\mathrm{ram}}(r)}{P_{\mathrm{ISM}}}\right)^{1 / 2} r
$$

where $r$ is a given radius where the wind has reached its terminal velocity. $P_{\mathrm{ISM}}=m n_{\mathrm{ISM}} v_{\mathrm{ISM}}^{2}$, with $n_{\mathrm{ISM}}$ and $v_{\mathrm{ISM}}$ being the total number density and velocity of the ISM adjacent to the stellar system. The proton mass is denoted by $m$. We use the same ISM neutral hydrogen density $\left(n_{\mathrm{ISM}}(\mathrm{HI})=\right.$ $\left.0.14 \mathrm{~cm}^{-3}\right)$ and proton density $\left(n_{\mathrm{ISM}}\left(\mathrm{H}^{+}\right)=0.1 \mathrm{~cm}^{-3}\right)$ as given by Model 10 in Table 1 of Wood et al. (2000) that are relevant for nearby systems in the local ISM to derive the total ISM number density ${ }^{2}$. The ISM velocity that each star sees represents the combined stellar motion and local interstellar cloud flow. The same observationally inferred ISM velocities are used for our sample as quoted in Wood et al. (2002, 2005, 2014, and references therein), given in our Table 1. The astrospheric radii that we derive are given in Table 2 . The variation in astrospheric sizes is quite large, ranging from 17 - $2760 \mathrm{au}$. In comparison, the heliosphere is approximately 122 au in radius (as observed by Voyager 1, Krimigis et al. 2013; Stone et al. 2019). $R_{\text {ast }}$ is used as a fixed outer spatial boundary condition for the cosmic ray transport model.

\section{COSMIC RAY MODEL}

We use a 1D diffusive-advection transport equation for the propagation of the Galactic cosmic rays. The model is described in detail in Rodgers-Lee et al. (2020b). However, we include a brief description here for completeness. Parker (1965) used the diffusive-advection transport equation in the context of the modulation of Galactic cosmic rays in the solar system. The $1 \mathrm{D}$ transport equation is given by

$$
\frac{\partial f}{\partial t}=\nabla \cdot(\kappa \nabla f)-v \cdot \nabla f+\frac{1}{3}(\nabla \cdot v) \frac{\partial f}{\partial \ln p}
$$

where $f, \kappa(p, B(r))$ and $p$ are the cosmic ray phase space density, spatial diffusion coefficient and momentum, respectively. $v(r)$ is the stellar wind velocity obtained from the stellar wind model. The first term on the righthand side of Eq. (2) accounts for the spatial diffusion of Galactic cosmic rays into the stellar system. The second and third terms act to suppress the flux of Galactic cosmic rays in different ways. The second term represents how the stellar wind advects the cosmic rays back out of the system, opposing their inward diffusion. The third term represents adiabatic losses due to the expansion of the stellar wind. This shifts the cosmic rays to lower energies. Eq. 2 does not account for cosmic ray drift motions (Jokipii et al. 1977). As noted in Rodgers-Lee et al. (2020b) this means that latitudinal variations and temporal variation of Galactic cosmic ray modulation due to a stellar activity cycle cannot be studied here. $2 \mathrm{D}$ or $3 \mathrm{D}$ cosmic ray transport models (such as Cohen et al. 2012; Potgieter et al. 2015) are needed to include these effects.

2 For $\pi^{1}$ UMa, the values given in Section 2 are used (from Wood et al. 2014). 
Table 1. The stellar properties for the five stars considered are listed here. The columns are: the name of the star, other identifiers for the stars, spectral type, age, mass, radius, rotation period, rotation rate in terms of the present-day solar value $\left(\Omega_{\odot}=2.67 \times 10^{-6}\right.$ rad s $\left.{ }^{-1}\right)$, x-ray luminosity, average unsigned radial surface magnetic field strength, mass-loss rate from Wood et al. (2002, 2005, 2014) in terms of the present-day value $\left(\dot{M}_{\odot}=2 \times 10^{-14} \mathrm{M}_{\odot} \mathrm{yr}^{-1}\right)$, ISM velocity, distance to each star and whether an exoplanet has been detected for each system, respectively. References for each property are also given.

\begin{tabular}{|c|c|c|c|c|c|c|c|c|c|c|c|c|c|}
\hline Star & Other identifier & Spec. type & $t_{\star}$ & $M_{\star}$ & $R_{\star}$ & $P_{\text {rot }}$ & $\Omega_{\star}$ & $\log L_{\mathrm{X}}$ & $\left|B_{\star}\right|$ & $\dot{M}_{\mathrm{W}}$ & $v_{\text {ISM }}$ & $d$ & $\begin{array}{c}\text { Known } \\
\text { Exoplanet? }\end{array}$ \\
\hline & & & [Gyr] & {$\left[M_{\odot}\right]$} & {$\left[R_{\odot}\right]$} & [day] & {$[\Omega \odot]$} & {$\left[\operatorname{erg~s}^{-1}\right]$} & {$[\mathrm{G}]$} & {$\left[\dot{M}_{\odot}\right]$} & {$\left[\mathrm{km} \mathrm{s}^{-1}\right]$} & {$[\mathrm{pc}]$} & \\
\hline 61 Cyg A & HD 201091A & $\mathrm{K} 5 \mathrm{~V}^{a}$ & $3.6^{a}$ & $0.66^{a}$ & $0.62^{a}$ & $34.2^{a}$ & 0.80 & $28.22^{a}$ & $3.8^{c}$ & $0.5^{b}$ & $86^{b}$ & $3.5^{b}$ & No \\
\hline$\epsilon$ Eri & HD 22049 & $\mathrm{~K} 2 \mathrm{~V}^{a}$ & $0.4-0.8^{a, d, e}$ & $0.86^{a}$ & $0.77^{a}$ & $10.3^{a}$ & 2.6 & $28.32^{a}$ & $8.1^{a}$ & $30.0^{b}$ & $27^{b}$ & $3.2^{b}$ & Yes \\
\hline$\epsilon$ Ind & HD 209100 & $\mathrm{~K} 5 \mathrm{~V}^{f}$ & $3.7-5.7^{g}$ & $0.75^{g}$ & $0.75^{f}$ & $37.2^{f}$ & 0.73 & $27.39^{f}$ & $17.6^{f}$ & $0.5^{b}$ & $68^{b}$ & $3.6^{b}$ & Yes \\
\hline$\pi^{1} \mathrm{UMa}$ & HD 72905 & $\mathrm{G} 1.5 \mathrm{~V}^{a}$ & $0.5^{a}$ & $1.00^{a}$ & $1.00^{a}$ & $5.00^{a}$ & 5.4 & $28.97^{a}$ & $7.6^{a}$ & $0.5^{h}$ & $34^{h}$ & $14^{h}$ & No \\
\hline$\xi$ Boo B & HD 131156B & $\mathrm{K} 4 \mathrm{~V}^{a}$ & $2^{a}$ & $0.99^{a}$ & $1.07^{a}$ & $10.3^{a}$ & 2.64 & $27.97^{a}$ & $11.8^{a}$ & $4.5^{i}$ & $32^{i}$ & $6.70^{i}$ & No \\
\hline
\end{tabular}

(a) Vidotto et al. (2014); (b) Wood et al. (2002); (c) Boro Saikia et al. (2016); (d) Mamajek \& Hillenbrand (2008); (e) Mawet et al. (2019); (f) Vidotto et al. (2016); (g) Feng et al. (2019); (h) Wood et al. (2014); (i) Wood et al. (2005).

Table 2. Stellar wind properties and other parameters relevant for the cosmic ray simulations. The columns are: the star's name, the stellar wind terminal velocity, the mass-loss rate obtained using the Weber-Davis model, the astrospheric size, the ISM ram pressure, the habitable zone (HZ) location and the wind base temperature, respectively.

\begin{tabular}{lcccccc}
\hline Star & $v_{\infty}$ & $\dot{M}_{\mathrm{WD}}$ & $R_{\text {ast }}$ & $P_{\mathrm{ISM}}$ & $\mathrm{HZ}$ & $T_{\text {base }}$ \\
\hline & {$\left[\mathrm{km} \mathrm{s}^{-1}\right]$} & {$\left[\dot{M}_{\left.\odot \mathrm{yr}^{-1}\right]}\right]$} & {$[\mathrm{au}]$} & {$\left[\mathrm{g} \mathrm{cm}^{-1} \mathrm{~s}^{-2}\right]$} & {$[\mathrm{au}]$} & {$[\mathrm{MK}]$} \\
\hline 61 Cyg A & 840 & 0.2 & 17 & $3 \times 10^{-11}$ & $0.3-0.8$ & 2.7 \\
$\epsilon$ Eri & 910 & 15 & 430 & $3 \times 10^{-12}$ & $0.5-1.1$ & 2.6 \\
$\epsilon$ Ind & 580 & 0.3 & 22 & $2 \times 10^{-11}$ & $0.4-0.9$ & 1.5 \\
$\xi$ Boo B & 770 & 2.7 & 140 & $4 \times 10^{-12}$ & $0.5-1.3$ & 1.7 \\
$\pi^{1} \mathrm{UMa}$ & 1380 & 0.2 & 44 & $5 \times 10^{-12}$ & $0.8-1.8$ & 3.3 \\
\hline$\pi^{1} \mathrm{UMa}$ & 1050 & 150 & 2760 & $5 \times 10^{-12}$ & $0.8-1.8$ & 3.3 \\
\hline
\end{tabular}

The diffusion coefficient, $\kappa$, for the cosmic rays depends on the level of turbulence in the magnetic field and on their momentum. Based on quasi-linear theory (Jokipii 1966; Schlickeiser 1989), $\kappa$ can be expressed as

$$
\frac{\kappa(p, B(r))}{\beta c}=\eta_{0}\left(\frac{p}{p_{0}}\right)^{1-\gamma} r_{\mathrm{L}}
$$

where $r_{\mathrm{L}}$ is the Larmor radius of the cosmic rays and $p_{0}=$ $3 \mathrm{GeV} / c$. Eq. 3 assumes isotropic diffusion. In reality, there are different components for parallel, $\kappa_{\|}$, and perpendicular, $\kappa_{\perp}$, diffusion with respect to the magnetic field direction. Similar to Herbst et al. (2020), as a first approximation we have assumed in Eq. 3 that $\kappa=\beta \lambda / 3$ where the particle mean free path, $\lambda$, scales with $r_{\mathrm{L}}$. A more complete description of the diffusion coefficient requires more information than is currently available about the type of turbulence present in these systems. The power law index $\gamma$ represents the type of turbulence present, such as Bohm diffusion $(\gamma=1)$, Kolmogorov-type turbulence $(\gamma=5 / 3)$ or magnetohydrodynamic-driven turbulence $(\gamma=3 / 2)$. Here we adopt $\gamma=1$, similar to Rodgers-Lee et al. (2020b). $\eta_{0}$ relates to the level of turbulence in the magnetic field (see Eq.(4) from Rodgers-Lee et al. 2020b). For the cosmic ray simulation of the solar system we use $R_{\text {ast }}=122$ au (available from Voyager observations) and $\eta_{0}=1$. There are currently no observational constraints for the type or level of turbulence present in other stellar systems. Constraining the chemical abundances of fingerprint ions in exoplanetary atmospheres could provide constraints on the incident cosmic ray spectrum. This can potentially be used to infer the type/level of turbulence present. In this case, the exoplanetary atmosphere and host star properties need to be well-constrained. It is possible that there is an increased level of turbulence in young systems due to increased stellar activity leading to more coronal mass ejections (CMEs). CMEs are thought to drive turbulence in the solar wind (Cranmer 2017). However, simulations suggest that the strong magnetic fields of young stars may confine CMEs (Alvarado-Gómez et al. 2018). Overall, it is unclear how the values of $\gamma$ and $\eta_{0}$ would change for systems of different ages. We adopt $\gamma=1$ and $\eta_{0}=1$ for all of our simulations for simplicity (similar to Svensmark 2006; Cohen et al. 2012). It is important to note that adopting different values for $\gamma$ and $\eta_{0}$ would affect the results presented here.

For the boundary conditions, the inner spatial boundary condition is reflective. The outer spatial boundary condition is a fixed boundary condition which is set to be the LIS values using the model fit to the Voyager 1 data given in Eq. (1) of Vos \& Potgieter (2015). The inner and outer momentum boundary conditions are both outflow boundary conditions. The spatial and momentum grids are logarithmically spaced. The spatial grid ranges from 0.01 au to $R_{\text {ast }}$ for each star (given in Table 2). The momentum grid ranges from $0.15-$ $100 \mathrm{GeV} / c$ with the number of momentum bins, $N_{\mathrm{p}}=60$.

\section{RESULTS}

\subsection{Galactic cosmic ray intensities in the habitable zones}

The flux of Galactic cosmic rays in the habitable zone (shaded regions) for our sample is shown as a function of cosmic ray kinetic energy in Fig. 1. The habitable zones for the stars are calculated using Eq. (4) and (5) from Kopparapu et al. (2014) with the recent Venus and early Mars criteria valid for planetary masses between $0.1 M_{\oplus} \leqslant M_{p} \leqslant 5 M_{\oplus}$ (the values are given in Table 2). The grey dashed line indicates typical Galactic cosmic ray fluxes at Earth for comparison. The solid 
black line shows the LIS fit which is the fixed outer boundary condition. Fig. 1 includes the two different scenarios for $\pi^{1}$ UMa, assuming $\dot{M}_{\mathrm{W}}=0.5 \dot{M}_{\odot}$ (corresponding to $\dot{M}_{\mathrm{WD}}=$ $0.2 \dot{M}_{\odot}$, grey shaded region) inferred from the observations of Wood et al. (2014) and $M_{\mathrm{WD}}=150 \dot{M}_{\odot}$ (magenta shaded region) based on the extrapolation of the relation between solar flares and CMEs from Drake et al. (2013).

61 Cyg A is the only system with similar Galactic cosmic ray fluxes in the habitable zone as observed at Earth (by comparing the blue shaded region and grey dashed line in Fig. 1). The four other systems have much lower Galactic cosmic ray fluxes in their habitable zones. At $\mathrm{GeV}$ energies, there are approximately 6 orders of magnitude difference at $\mathrm{GeV}$ cosmic ray energies between the two most extreme systems (61 Cyg A and $\pi^{1}$ UMa assuming $\left.\dot{M}_{\mathrm{WD}}=150 \dot{M}_{\odot}\right)$. For $\pi^{1} \mathrm{UMa}$, the main result of adopting a different $\dot{M}$ (along with different ISM densities) for the cosmic ray transport model is the astrospheric size. For $\dot{M}_{\mathrm{WD}}=0.2 \dot{M}_{\odot}, R_{\text {ast }}=44 \mathrm{au}$, whereas for $\dot{M}_{\mathrm{WD}}=150 \dot{M}_{\odot}$, then $R_{\text {ast }}=2760$ au, i.e. 63 times bigger. At $\mathrm{GeV}$ energies, this changes the Galactic cosmic ray fluxes in the habitable zone by approximately 2 orders of magnitude (the grey and magenta shaded regions in Fig. 1). The kinetic energy at which the peak cosmic ray flux occurs also shifts from $4 \mathrm{GeV}$ to $8 \mathrm{GeV}$. Thus, adopting a different $\dot{M}$ and ISM densities for $\pi^{1}$ UMa significantly affects the Galactic cosmic ray fluxes in the habitable zone.

The habitable zones shown in Fig. 1 are at different orbital distances due to the stars' different spectral types and luminosities (shown in Table 2). We compared the Galactic cosmic ray fluxes at 1 au for the different systems. The general behaviour was very similar to what is seen in Fig. 1 with 61 Cyg A having the largest fluxes and $\pi^{1}$ UMa showing the smallest. However, this behaviour would not necessarily be expected at all orbital distances.

In order to understand the level of modulation suffered by the Galactic cosmic rays for the different systems it is necessary to consider two factors, namely the timescales for the different physical processes and the astrospheric size. Individual stellar properties, such as $B_{\star}$ or $\Omega_{\star}$ for instance, cannot be used directly to understand the Galactic cosmic ray modulation for the systems. The diffusive and advective timescales are given by

$$
t_{\mathrm{dif}}=\frac{r^{2}}{\kappa(p, B(r))}, \quad t_{\mathrm{adv}}=\frac{r}{v(r)} .
$$

If $t_{\mathrm{dif}}<t_{\mathrm{adv}}$ for a given cosmic ray energy then diffusion dominates. It means that the cosmic rays of that energy can diffuse into the system faster than the advective processes can act to suppress the Galactic cosmic rays. Fig. 2 shows the timescales as a function of orbital distance for two systems representative of the sample: $61 \mathrm{Cyg} \mathrm{A}$ and $\pi^{1} \mathrm{UMa}\left(\dot{M}_{\mathrm{WD}}=\right.$ $0.2 \dot{M}_{\odot}$ case). The diffusion timescale depends on momentum and so timescales for different cosmic ray energies are shown.

We find that the ratio of $t_{\text {dif }}$ for $\mathrm{GeV}$ energy cosmic rays (solid blue line) versus $t_{\text {adv }}$ (dashed red line) at large orbital distances, where the wind has reached its' terminal velocity and $B_{\phi}$ dominates, is a good indicator of the overall level of modulation that Galactic cosmic rays will suffer from. At these distances, $t_{\mathrm{dif}} / t_{\mathrm{adv}}$ remains constant with orbital distance because $t_{\mathrm{dif}} \propto r^{2} B_{\phi} \propto r$ and $t_{\mathrm{adv}} \propto r$.

For example, for $61 \mathrm{Cyg} \mathrm{A}$ at $1 \mathrm{GeV}$ and $r=R_{\text {ast }}$ in Fig. $2(\mathrm{a}), t_{\mathrm{dif}} / t_{\mathrm{adv}} \sim 0.1$ meaning that diffusion dominates and the cosmic ray fluxes are not greatly suppressed (indicated by the blue shaded region). Advective processes only dominate at $r \lesssim 0.2$ au for $\lesssim \mathrm{GeV}$ cosmic ray energies (indicated by the red shaded region). The opposite extreme is $\pi^{1}$ UMa, where $t_{\mathrm{dif}} / t_{\mathrm{adv}} \sim 4$ for $\mathrm{GeV}$ energies at $r=R_{\text {ast }}$ (irrespective of the $\dot{M}$ adopted since $v_{\infty}$ and $B(r)$ are very similar for both cases) and the cosmic ray fluxes are significantly suppressed, shown in Fig. 2(b). Advective processes dominate throughout the wind for $\pi^{1} \mathrm{UMa}$ at $\lesssim \mathrm{GeV}$ cosmic ray energies (indicated by the red shaded region). This is why for the larger $\dot{M}$ (or larger astrospheric radius) case, we see significantly more suppression of the Galactic cosmic ray fluxes. If instead diffusion dominated at large orbital distances changing the size of $R_{\text {ast }}$ would make little difference to the Galactic cosmic ray fluxes in the habitable zone, as found in Mesquita et al. (2021b) for the GJ 436 system.

It is likely that there will be variations from this 'rule' that we have proposed here. For instance, as we have discussed the size of $R_{\text {ast }}$ for advection-dominated systems affects the modulation of Galactic cosmic rays. $R_{\text {ast }}$ depends on the ISM properties. This is not a property intrinsic to the star itself and thus, variation from star to star should be expected. Another property that may lead to deviations from our rule is $\Omega_{\star}$. We are relying on the value of $t_{\mathrm{dif}} / t_{\mathrm{adv}}$ at large orbital distances to guide us, i.e. where the wind has reached its' terminal velocity and therefore the ratio $t_{\text {dif }} / t_{\mathrm{adv}}$ is constant with orbital distance. However, at large orbital distances the magnetic field changes from being dominated by the radial component to being dominated by the azimuthal component, i.e. the tightening of the Parker spiral. This occurs for the stars in our sample at approximately 1 au which is also approximately where the habitable zone for the stars is located. This means that $t_{\text {dif }} / t_{\mathrm{adv}}$ also changes in this region, generally becoming more advection-dominated. Thus, the location of this turn-over point will affect the modulation of Galactic cosmic rays (see Mesquita et al. 2021a, for a more detailed discussion of this effect).

\subsection{Galactic cosmic ray intensities at planets' orbits}

For the two systems which host known exoplanets $(\epsilon$ Eri and $\epsilon$ Ind), the Galactic cosmic ray flux at the orbital distance of the planets is shown in Fig. 3. Again, for comparison we overplot the LIS and fluxes at Earth, denoted by the solid black line and dashed grey line, respectively. For $\epsilon$ Ind b, an approximately $\sim 3$ Jupiter mass planet orbiting at $11.55 \mathrm{au}$, it receives larger Galactic cosmic ray fluxes than Earth. On the other hand, the fluxes reaching $\epsilon$ Eri b, an approximately Jupiter mass planet orbiting at $3.39 \mathrm{au}$, are much lower.

Mawet et al. (2019) suggested that $\epsilon$ Erib should be detected via direct imaging with JWST's NIRCam instrument, depending on model assumptions for the exoplanet. They also discussed that spectroscopic age indicators suggest that the star is closer to $800 \mathrm{Myr}$ old, rather than $200 \mathrm{Myr}$. Therefore, they indicated the planet will have an effective temperature of $\sim 150 \mathrm{~K}$. Spectroscopic observations with JWST's NIRSpec instrument could additionally probe molecular abundances in the upper atmosphere of the planet. Modelling of the chemical impact of Galactic cosmic rays in $\epsilon$ Eri b could investigate whether there are any signatures that will be observable with JWST. This could be used to constrain our models in the future. $\epsilon$ Ind is thought to be an older system and therefore 


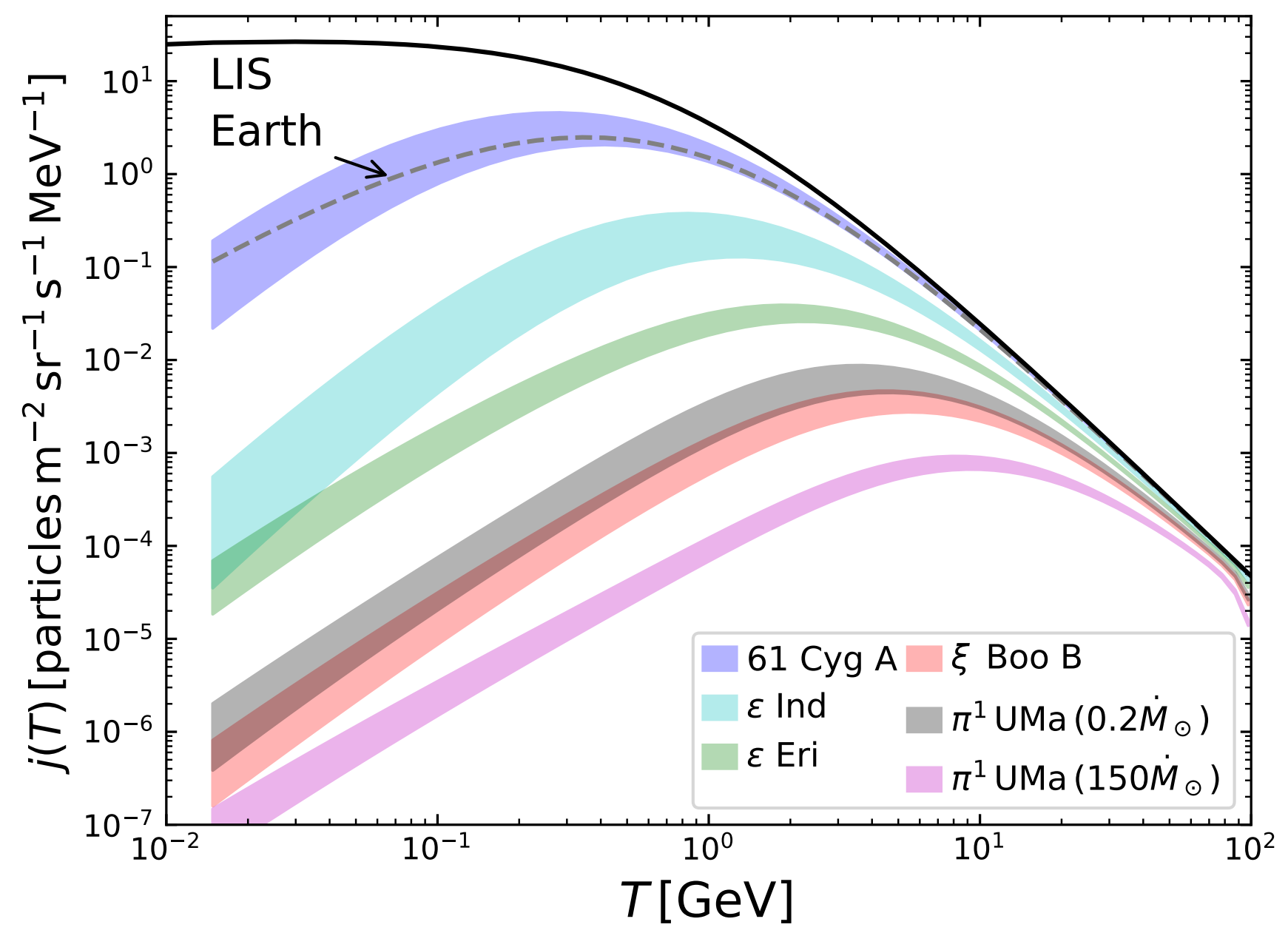

Figure 1. The differential intensities of Galactic cosmic rays in the habitable zone are shown by the shaded regions for each star in the sample as a function of cosmic ray kinetic energy. The solid black line represents the LIS. The grey dashed line shows the differential intensities at Earth for comparison.

it is less likely that the exoplanet would be detectable with JWST (Viswanath et al. 2021).

A number of studies have previously focused on $\mathrm{M}$ dwarf systems, some of which host known exoplanets (Herbst et al. 2020; Mesquita et al. 2021b,a). Herbst et al. (2020) use 3D magneto-hydrodynamic simulations to model the interaction of the stellar wind with the ISM for $3 \mathrm{M}$ dwarf systems. These simulations give more information about the stellar equivalent of the heliosheath and the termination shock which we do not account for in our model. However, in comparison to Herbst et al. (2020), local ISM properties were available for all of the stars in our sample which is important for advectiondominated systems. Mesquita et al. (2021b) used similar ISM observational constraints for GJ 436 with an estimate for its stellar magnetic field strength. Thus, our sample represents the most well-constrained sample. Mesquita et al. (2021a) perform a complementary study considering nearby $M$ dwarf systems (using a similar selection criteria as for our sample).

Herbst et al. (2020) found significant Galactic cosmic ray fluxes for Proxima Cen b and LHS $1140 \mathrm{~b}$. In comparison, Mesquita et al. (2021b) found much lower fluxes reaching GJ
$436 \mathrm{~b}$ (although it is important to note that GJ $436 \mathrm{~b}$ orbits much closer in than the habitable zone). Some of the variety of behaviour found between these two studies can be attributed to different cosmic ray transport properties (i.e. Kolmogorov-type turbulence versus Bohm diffusion). However, despite this, a variety of behaviour is observed due to the different stellar wind properties and astrospheric sizes (which affects advection-dominated systems). Thus, while it is now evident that Galactic cosmic rays are an important ingredient to consider for exoplanet habitability, it will hopefully also be possible with JWST observations of exoplanetary atmospheres to constrain our models. The sample from Mesquita et al. (2021a) includes 8 known exoplanets, presenting an exciting opportunity for upcoming observations. The energetic particle flux at a given height in an exoplanetary atmosphere can be constrained by observations that probe the abundances of fingerprint ions, such as $\mathrm{H}_{3} \mathrm{O}^{+}$and $\mathrm{NH}_{4}^{+}$ (Barth et al. 2021). In theory, the molecular bands for $\mathrm{H}_{3} \mathrm{O}^{+}$ are within the spectral range of JWST's NIRSpec instrument (e.g. Bourgalais et al. 2020). Thus, future 3D magnetohydrodynamic simulations of stellar winds, (such as those 


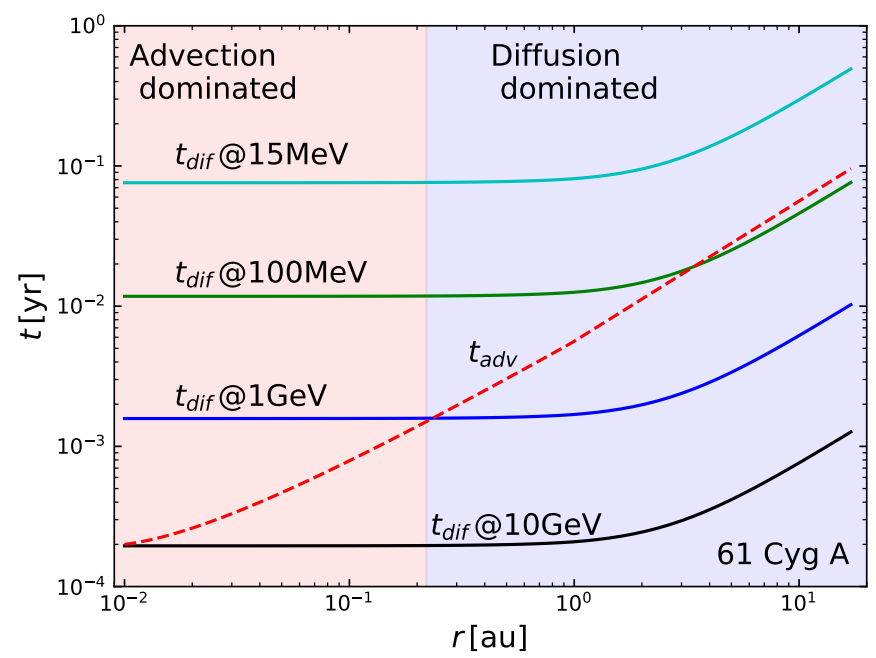

(a) 61 Cyg A

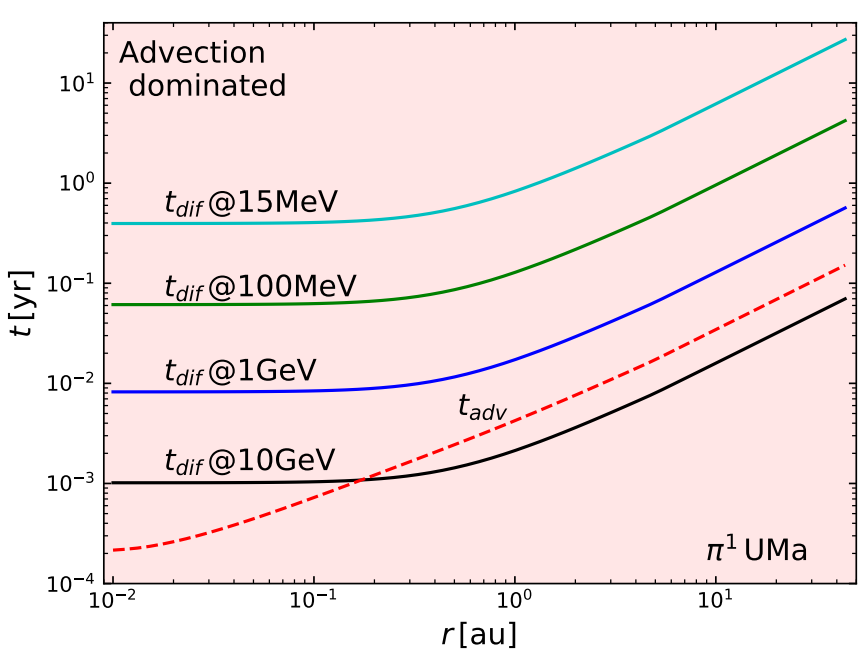

(b) $\pi^{1} \mathrm{UMa}$

Figure 2. The diffusion timescales for different cosmic ray energies (solid lines) and the advective timescale (red dashed line) as a function of orbital distance are shown for two systems representative of the sample that we consider. The blue shaded region in (a) for the 61 Cyg A system represents a "diffusion-dominated" region where $t_{\mathrm{dif}}<t_{\mathrm{adv}}$ for GeV energy cosmic rays. The red shaded regions in (a) and (b) represent "advection-dominated" regions where $t_{\mathrm{dif}}>t_{\mathrm{adv}}$ for $\mathrm{GeV}$ energy cosmic rays.



Figure 3. The Galactic cosmic ray fluxes for the planets in our sample, $\epsilon$ Erib (solid green line) and $\epsilon$ Ind b (solid cyan line). The solid black line represents the LIS. The grey dashed line shows the differential intensities at Earth for comparison.

presented in Herbst et al. 2020), combined with 2D/3D cosmic ray transport models appear well motivated.

\section{DISCUSSION \& CONCLUSIONS}

We investigated the suppression, or modulation, of Galactic cosmic rays for 5 nearby, well-studied solar-type stars. We modelled the propagation of Galactic cosmic rays through the stellar wind using a combined stellar wind and cosmic ray transport model. The stellar wind properties are well constrained as both mass-loss rates inferred from observations, stellar magnetic field measurements and ISM velocities are available in the literature for our sample. This represents the most well-constrained sample presented to date and offers valuable physical insight with regard to the importance of the ISM velocities for certain systems. Two of the stars in the sample host known Jupiter-like exoplanets.

We found that the Galactic cosmic ray fluxes in the habitable zone for four of the five stars were significantly lower than the fluxes received at Earth. $61 \mathrm{Cyg} \mathrm{A}$ is the only system with comparable fluxes to Earth in its' habitable zone. $61 \mathrm{Cyg} \mathrm{A}$ is an interesting system because it displays a solarlike magnetic cycle (Boro Saikia et al. 2016) with polarity reversals in the large-scale magnetic geometry. Here we did not investigate the effect of such a magnetic cycle on the modulation of Galactic cosmic rays, but similar to the solar cycle it would most likely introduce cyclic variations in the Galactic cosmic ray fluxes. The drift of cosmic rays would be interesting to study for $61 \mathrm{Cyg} \mathrm{A}$ which would require more complex modelling than presented here. In addition, $\epsilon$ Ind b, orbiting at $11.55 \mathrm{au}$, received higher fluxes than Earth while the other known exoplanet $\epsilon$ Erib is exposed to much lower fluxes.

We identify two important regimes displayed by the systems in our study. When the diffusion timescale for $\mathrm{GeV}$ energy cosmic rays is shorter than the advective timescale (diffusion-dominated) at large orbital distances we find that (a) the Galactic cosmic rays are least suppressed in these systems and (b) varying the astrospheric size would not change the level of suppression significantly (similar to what was found by Mesquita et al. 2021b, for the M dwarf GJ436). $61 \mathrm{Cyg} \mathrm{A}$ is one such system. On the other hand, when the advection timescale is shorter than the diffusion timescale (advection-dominated) we find the opposite: the Galactic cosmic rays are most suppressed in these systems and varying the astrospheric size also changes the level of suppression significantly. $\pi^{1} \mathrm{UMa}$ is an example of an advection-dominated system. Overall, this means that for any diffusion-dominated 
system knowledge about the astrospheric size (via the ISM properties) is not hugely important. For these systems having a well-constrained stellar wind model will provide sufficient information to robustly estimate the modulation of Galactic cosmic rays. Generally, diffusion-dominated systems are likely to be older due to slower terminal velocities and weaker stellar magnetic fields. It is important to note that our results depend on the cosmic ray transport properties that we adopt. Different cosmic ray transport properties would likely alter our results. A detection of emission from fingerprint ions, such as $\mathrm{H}_{3} \mathrm{O}^{+}$and $\mathrm{NH}_{4}^{+}$, from an exoplanetary atmosphere will constrain the incident energetic particle flux which will in turn could constrain the energetic particle transport properties. However, there will also be some dependence on the exoplanet atmosphere properties, such as the chemical composition. Exoplanets orbiting at different distances can be used to disentangle stellar radiation and stellar energetic particle effects from Galactic cosmic rays.

Our results show a wide range of Galactic cosmic ray fluxes in the habitable zone for the stars in our sample. While there are no known Earth-like planets in our sample we can speculate how Galactic cosmic ray fluxes could impact life on an Earth-like planet in the habitable zones. It is very challenging to detect Earth-like planets orbiting in the habitable zone of solar-type stars. Thus, it is entirely possible that undetected Earth-like planets exist in the habitable zone of our sample of stars. The radiation dose can be used as a measure of how damaging ionising radiation (e.g. cosmic rays or their secondary particles) is for cell tissue (see Atri 2020; Atri et al. 2020, for example). Atri (2017) indicated that the total atmospheric column depth is the most important quantity for determining the cosmic ray flux reaching the exoplanet's surface, rather than the chemical composition of the atmosphere, for instance. Thus, we can infer that the radiation dose received at the surface of an Earth-like planet in the habitable zone of the stars in our sample will be comparable, or lower, than the radiation dose received on the surface of Earth ( 0.6 mSv/day, Atri et al. 2020). This is because the Galactic cosmic rays fluxes in the habitable zones are comparable (for $61 \mathrm{Cyg}$ A) or lower than those reaching Earth for our sample. This is based on the assumption that any hypothetical Earth-like planet has a similar atmospheric column density to Earth and a similar planetary magnetic field strength. A stronger planetary magnetic field would prevent higher energy cosmic rays reaching the surface of the Earthlike planet (Grießmeier et al. 2015). Overall, this suggests that life as we know it would not be negatively impacted by the Galactic cosmic ray fluxes that we find for our sample.

\section{ACKNOWLEDGEMENTS}

We would like to thank the anonymous referee for helpful comments which improved the manuscript. DRL would like to thank Carolina Villarreal-d'Angelo for very helpful discussions which improved the paper. The authors acknowledge funding from the European Research Council (ERC) under the European Union's Horizon 2020 research and innovation programme (grant agreement No 817540, ASTROFLOW). The authors wish to acknowledge the Irish Centre for HighEnd Computing (ICHEC) for the provision of computational facilities and support. AAV and ALM acknowledge funding from the Provost's PhD Project award.

\section{DATA AVAILABILITY}

The output data underlying this article will be available via zenodo.org upon publication.

\section{APPENDIX A: STELLAR WIND PROFILES}

The stellar wind profiles that are used in the cosmic ray transport simulations for our sample of stars are shown in Fig.A1. The left axis gives the magnetic field strength (total, radial and azimuthal magnetic field strengths shown by solid blue, green and red lines, respectively) and the right axis shows the velocity (dashed magenta lines) as a function of orbital distance. The stellar wind model extends from the base of the wind at $1 R_{\star}$ to an orbital distance where the stellar wind has reached its terminal velocity, at 1 au for the solar system, for instance. Therefore, beyond this distance we extrapolate the stellar wind properties out to the size of each astrosphere for the cosmic ray transport model. The velocity is extrapolated as $v(r>1 \mathrm{au})=v(r=1 \mathrm{au})$. For the magnetic field, $B_{r}(r>1 \mathrm{au})=B_{r}(r=1 \mathrm{au}) / r^{2}$ and $B_{\phi}(r>1 \mathrm{au})=B_{\phi}(r=1 \mathrm{au}) / r$. The inner boundary for the cosmic ray transport model is at 0.01 au for all of the simulations. The distance at which the wind has reached its terminal velocity is slightly larger than $1 \mathrm{au}$ for some of the systems considered here which we account for when we extrapolate the data.

\section{REFERENCES}

Aharonian F., Peron G., Yang R., Casanova S., Zanin R., 2020, Phys. Rev. D, 101, 083018

Airapetian V. S., Glocer A., Gronoff G., Hébrard E., Danchi W., 2016, Nature Geoscience, 9, 452

Alvarado-Gómez J. D., Drake J. J., Cohen O., Moschou S. P., Garraffo C., 2018, ApJ, 862, 93

Atri D., 2017, MNRAS, 465, L34

Atri D., 2020, MNRAS, 492, L28

Atri D., MacArthur C., Dobbs-Dixon I., 2020, arXiv e-prints, p. arXiv:2012.00568

Barth P., et al., 2021, MNRAS, 502, 6201

Benedict G. F., et al., 2006, AJ, 132, 2206

Boro Saikia S., et al., 2016, A\&A, 594, A29

Bourgalais J., et al., 2020, ApJ, 895, 77

Chen H., Zhan Z., Youngblood A., Wolf E. T., Feinstein A. D., Horton D. E., 2020, Nature Astronomy, 5, 298

Cohen O., Drake J. J., Kóta J., 2012, ApJ, 760, 85

Cranmer S. R., 2017, ApJ, 840, 114

Cummings A. C., et al., 2016, ApJ, 831, 18

Dartnell L. R., 2011, Astrobiology, 11, 551

Drake J. J., Cohen O., Yashiro S., Gopalswamy N., 2013, ApJ, 764,170

Feng F., Anglada-Escudé G., Tuomi M., Jones H. R. A., Chanamé J., Butler P. R., Janson M., 2019, MNRAS, 490, 5002

Gardner J. P., et al., 2006, Space Sci. Rev., 123, 485

Globus N., Blandford R. D., 2020, ApJ, 895, L11

Globus N., Fedynitch A., Blandford R. D., 2021, ApJ, 910, 85

Grießmeier J. M., Tabataba-Vakili F., Stadelmann A., Grenfell J. L., Atri D., 2015, A\&A, 581, A44 


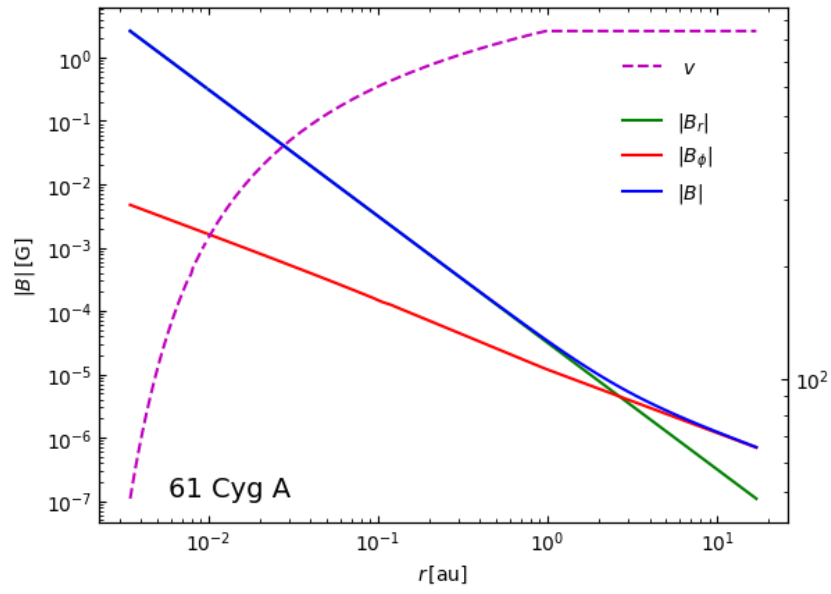

(a)

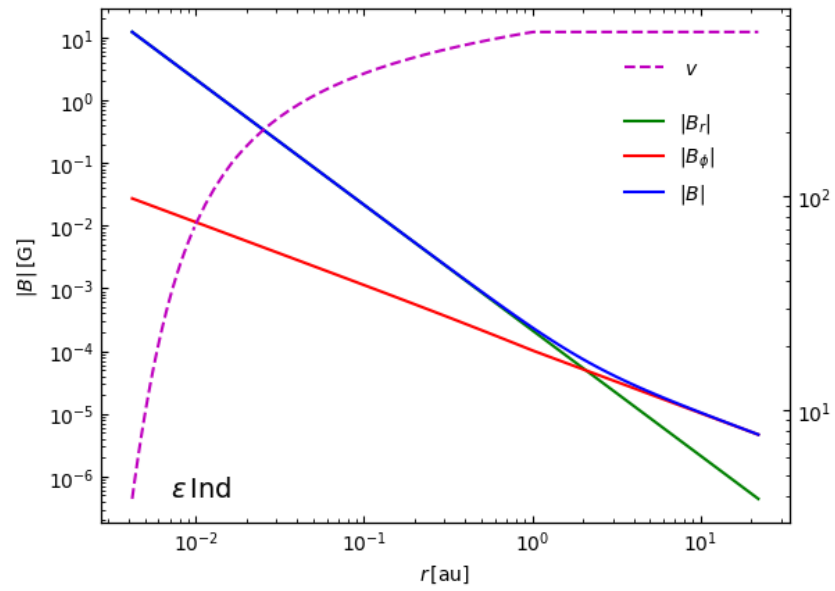

(c)

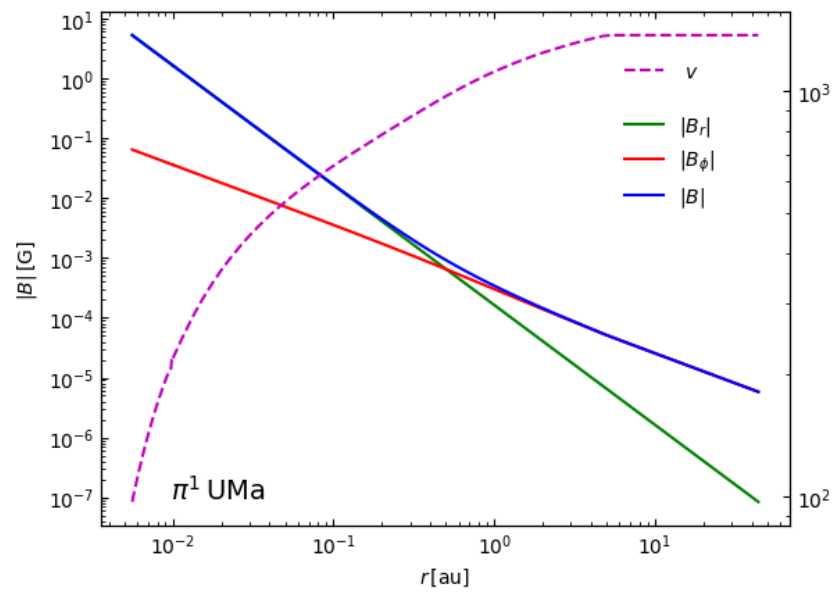

(e)

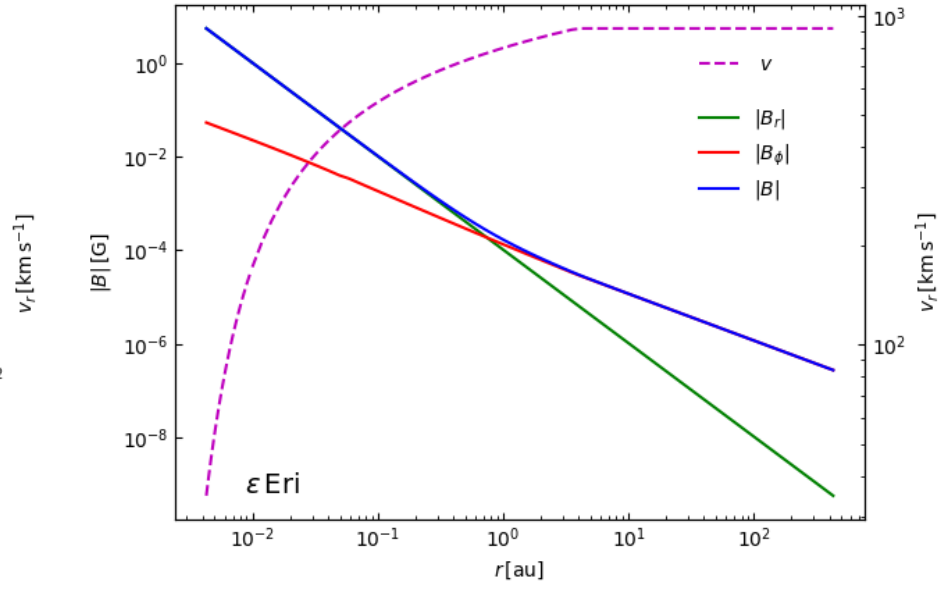

(b)

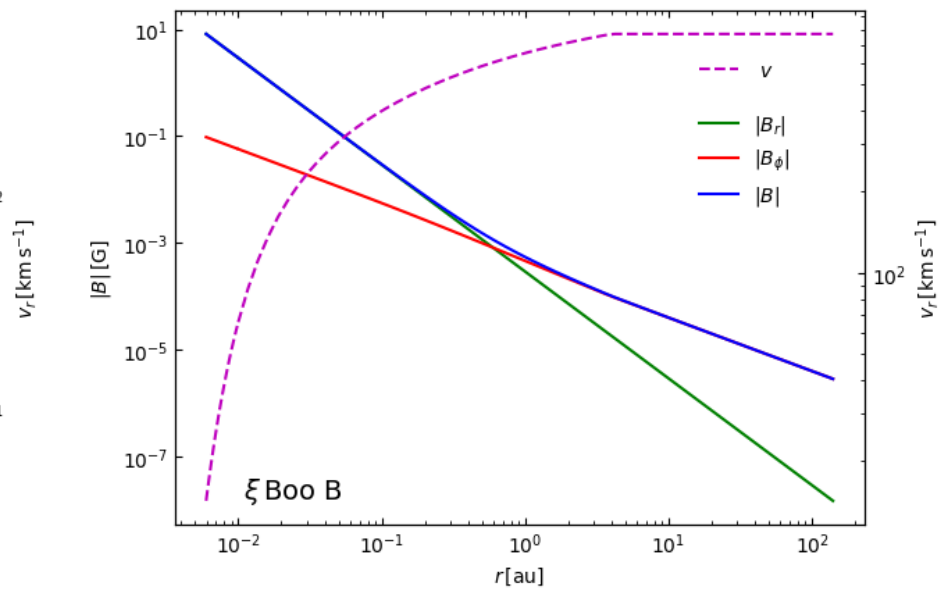

(d)

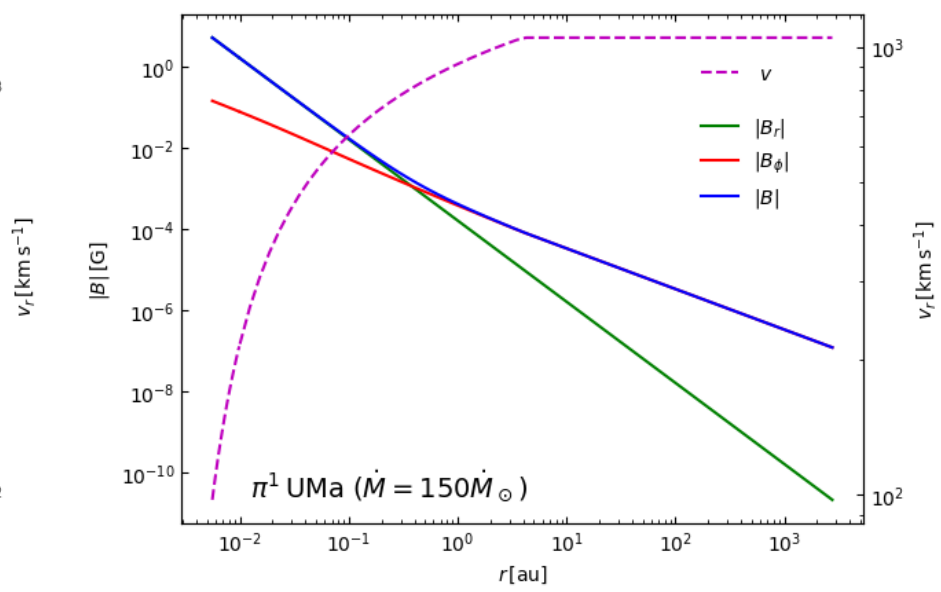

(f)

Figure A1. The magnetic field and velocity profiles are shown as a function of radial distance for the five stars in our sample. In each panel, the blue, green and red solid lines are the total, radial and azimuthal components of the stellar wind magnetic field, respectively. The dashed magenta line is the velocity profile of the stellar wind. The stellar wind model values extend to $\sim 1$ au depending on the system, beyond which the data is extrapolated as described in the text. The values used in the cosmic ray simulations start at 0.01 au. 
Hatzes A. P., et al., 2000, ApJ, 544, L145

Helling C., Rimmer P. B., 2019, Philosophical Transactions of the Royal Society of London Series A, 377, 20180398

Herbst K., et al., 2020, ApJ, 897, L27

Holzwarth V., Jardine M., 2007, A\&A, 463, 11

Johnstone C. P., 2017, A\&A, 598, A24

Johnstone C. P., Güdel M., 2015, A\&A, 578, A129

Johnstone C. P., Güdel M., Brott I., Lüftinger T., 2015, A\&A, 577, A28

Jokipii J. R., 1966, ApJ, 146, 480

Jokipii J. R., Levy E. H., Hubbard W. B., 1977, ApJ, 213, 861

Kopparapu R. K., Ramirez R. M., SchottelKotte J., Kasting J. F., Domagal-Goldman S., Eymet V., 2014, ApJ, 787, L29

Krimigis S. M., Decker R. B., Roelof E. C., Hill M. E., Armstrong T. P., Gloeckler G., Hamilton D. C., Lanzerotti L. J., 2013, Science, 341, 144

Mamajek E. E., Hillenbrand L. A., 2008, ApJ, 687, 1264

Mawet D., Hirsch L., Lee E. J., Ruffio J.-B., Bottom M., Fulton B. J., 2019, AJ, 157, 33

McComas D. J., Ebert R. W., Elliott H. A., Goldstein B. E., Gosling J. T., Schwadron N. A., Skoug R. M., 2008, Geophys. Res. Lett., 35, L18103

Mesquita A. L., Rodgers-Lee D., Vidotto A. A., Atri D., Wood B. W., 2021a, submitted to MNRAS

Mesquita A. L., Rodgers-Lee D., Vidotto A. A., 2021b, MNRAS, 505, 1817

Ó Fionnagáin D., Vidotto A. A., 2018, MNRAS, 476, 2465

Parker E. N., 1965, Planet. Space Sci., 13, 9

Pathak P., Petit dit de la Roche D. J. M., Kasper M., Sterzik M., Absil O., Boehle A., Feng F., 2021, arXiv e-prints, p. arXiv:2104.13032

Potgieter M. S., 2013, Living Reviews in Solar Physics, 10, 3

Potgieter M. S., Vos E. E., Munini R., Boezio M., Di Felice V., 2015, ApJ, 810, 141

Rab C., Güdel M., Padovani M., Kamp I., Thi W.-F., Woitke P., Aresu G., 2017, A\&A, 603, A96

Redfield S., Linsky J. L., 2000, ApJ, 534, 825

Rodgers-Lee D., Taylor A. M., Ray T. P., Downes T. P., 2017, MNRAS, 472, 26

Rodgers-Lee D., Taylor A. M., Downes T. P., Ray T. P., 2020a, MNRAS, 491, 4742

Rodgers-Lee D., Vidotto A. A., Taylor A. M., Rimmer P. B., Downes T. P., 2020b, MNRAS, 499, 2124

Rodgers-Lee D., Taylor A. M., Vidotto A. A., Downes T. P., 2021, MNRAS, 504, 1519

Schlickeiser R., 1989, ApJ, 336, 243

Stone E. C., Cummings A. C., Heikkila B. C., Lal N., 2019, Nature Astronomy, 3, 1013

Svensmark H., 2006, Astronomische Nachrichten, 327, 871

Tabataba-Vakili F., Grenfell J. L., Grießmeier J. M., Rauer H., 2016, A\&A, 585, A96

Vidotto A. A., 2021, Living Reviews in Solar Physics, 18, 3

Vidotto A. A., et al., 2014, MNRAS, 441, 2361

Vidotto A. A., et al., 2016, MNRAS, 455, L52

Viswanath G., Janson M., Dahlqvist C.-H., Petit dit de la Roche D., Samland M., 2021, arXiv e-prints, p. arXiv:2105.09773

Vos E. E., Potgieter M. S., 2015, ApJ, 815, 119

Weber E. J., Davis Leverett J., 1967, ApJ, 148, 217

Wood B. E., 2004, Living Reviews in Solar Physics, 1, 2

Wood B. E., Müller H.-R., Zank G. P., 2000, ApJ, 542, 493

Wood B. E., Müller H.-R., Zank G. P., Linsky J. L., 2002, ApJ, 574,412

Wood B. E., Redfield S., Linsky J. L., Müller H.-R., Zank G. P., 2005, ApJS, 159, 118

Wood B. E., Müller H.-R., Redfield S., Edelman E., 2014, ApJ, 781, L33

Wood B. E., et al., 2021, arXiv e-prints, p. arXiv:2105.00019 